\title{
Persistent, intermittent, and undetected accretion-powered millisecond $X$-ray pulsations from neutron stars in low-mass X-ray binary systems
}

\author{
Stratos Boutloukos ${ }^{\star a}$, Ka Ho Lo ${ }^{b}$, M. Coleman Miller ${ }^{c}$, and Frederick K. Lamb ${ }^{b d}$ \\ ${ }^{a}$ Institute for Astronomy and Astrophysics, University of Tübingen, Auf der Morgenstelle 10, \\ Tübingen, 72076, Germany \\ ${ }^{b}$ Center for Theoretical Astrophysics and Department of Physics, University of Illinois at \\ Urbana-Champaign, 1110 West Green Street, Urbana, IL 61801-3080, USA \\ ${ }^{c}$ University of Maryland, Department of Astronomy and Joint Space-Science Institute, College \\ Park, MD 20742-2421, USA \\ ${ }^{d}$ Department of Astronomy, University of Illinois at Urbana-Champaign, 1002 West Green Street, \\ Urbana, IL 61801-3074, USA \\ E-mail: stratos@tat.physik.uni-tuebingen.de
}

\begin{abstract}
Accretion-powered millisecond X-ray pulsations have been detected in only 13 of more than 130 known neutron stars in low-mass X-ray binary systems (LMXBs). Pulsations have been detected only intermittently in three of these accretion-powered millisecond X-ray pulsars. We show that the failure to detect accretion-powered pulsations from most neutron-star LMXBs, and the intermittent detection of pulsations in a few, can both be explained if the emitting regions of accreting neutron stars with millisecond spin periods are typically close to their spin poles and move in response to changes in the accretion flow. This nearly-aligned moving spot model is consistent with the small observed amplitudes and nearly sinusoidal waveforms of most accreting millisecond X-ray pulsars, and the large, rapid phase variations of several.
\end{abstract}

Fast $X$-ray timing and spectroscopy at extreme count rates

February 7-11, 2011

Champéry, Switzerland

\footnotetext{
${ }^{*}$ Speaker.
} 


\section{Introduction}

The unique capabilities of the Rossi X-ray Timing Explorer (RXTE) have led to the discovery of thirteen neutron stars in low-mass X-ray binary systems (LMXBs) that produce accretionpowered X-ray oscillations at their millisecond spin periods. Oscillations have been detected only intermittently from three of these accretion-powered millisecond X-ray pulsars (APMXPs), with duty cycles as low as $0.03 \%$ [4]. At present, accretion-powered pulsations have not been detected in the vast majority of more than 130 known neutron-star LMXBs in the Galaxy [10], including a dozen in which the neutron star spin rate is known from its nuclear-powered oscillations (see [7]).

The pulsations of APMXPs share several characteristics. Their rms amplitudes are usually only a few percent (although a few stars sometimes produce amplitudes as large as 19\% [13]) and the profiles of most are nearly sinusoidal (the rms amplitude of the first overtone of the spin frequency is typically less than $\sim 3 \%$ [15]). A notable exception is the profile of the recently discovered APMXP SWIFT J1749.4-2807, which sometimes has fundamental and first overtone amplitudes as large as $29 \%$ and $23 \%$ respectively [2]. In some stars, the pulse arrival time varies erratically by as much as 0.3 cycles [14]. The pulsations of the three intermittent APMXPs have amplitudes $<3 \%$ and are nearly sinusoidal (a first overtone has been detected only once, in an observation of HETE J1900.1-2455, with an amplitude of 0.4\% [6]). For comparison, persistent pulsations with amplitudes as low as $\sim 0.5 \%$ can be detected in some stars using RXTE; the upper limits on pulsation amplitudes in stars in which they have not been detected range up to $10 \%$.

APMXPs are thought to be weakly magnetic stars that have been spun up by accreting angular momentum (see [7] and references therein). The similarity between accretion-powered oscillations and burst oscillations in sources that show both [1] indicate that many other neutron stars in LMXBs have been spun up to millisecond spin periods. During the spin-up phase, the star's magnetic poles are thought to migrate toward its spin poles [5]. The magnetic poles of a star that has been spun up to a high spin frequency will therefore be close to its spin poles and accretion to the surface near the magnetic poles will therefore produce weak pulsations. In contrast, the magnetic poles (and hence the emitting regions) of a star that is spinning down because it has a low average accretion rate are likely to be farther from the spin poles, so its pulsations will be stronger. This is consistent with the low average accretion rates of all known APMXPs.

Several ideas have been proposed to explain the failure to detect pulsations in many neutron stars in LMXBs (see, e.g., [12]). Only the nearly aligned moving spot model [8] explains naturally the appearance and disappearance of pulsations on timescales as short as a few minutes. In this model, the closeness of the X-ray emitting areas to the spin axis means that small motions in response to variations in the structure and accretion rate of the disk can produce large changes in the observed amplitude and phase of the pulsations. Here we show that the model can also explain the non-detection of accretion-powered pulsations in most neutron-star LMXBs using current instruments. The reason is that emitting areas that are very close to the star's spin poles produce pulsations that are very weak. The much larger timing noise produced by movement of highlatitude emitting areas can further reduce the pulse amplitude. As a result, pulsations will only sporadically exceed the detection limit, when changes in the accretion flow cause the the emitting areas to move away from the spin poles by a few degrees. 


\section{Pulse profile modeling}

We used the ray-tracing code described in [8] to calculate the pulse profiles produced by emitting spots at various rotational latitudes when viewed at different inclinations. This code uses the Schwarzschild plus Doppler (S+D) approximation [11], which treats the special relativistic Doppler effects (such as aberrations and energy shifts) associated with the rotational motion of the stellar surface exactly, but treats the star as spherical and uses the Schwarzschild spacetime to compute the general relativistic redshift, trace the propagation of light from the stellar surface to the observer, and calculate light travel-time effects. For the stellar models we consider, rotational distortion of the star and frame dragging can be neglected [3]. The code has been validated by comparing its results with analytical results for special cases and with previously reported numerical results.

Pulse profiles are determined by folding X-ray data over time intervals long enough that the profiles are stable. The required intervals are typically $\sim 10^{5}-10^{6}$ times the $\sim 1 \mathrm{~ms}$ dynamical timescale near the neutron star. The emission and beaming patterns used in computing pulse profiles therefore represent averages of the patterns over time intervals like these. We assume radiation from the stellar surface reaches the observer without interacting with any intervening matter. Guided by the observations summarized in the Introduction, we assume that X-ray pulsations will be detected if the fractional amplitude of any harmonic is at least $0.5 \%$.

\section{Results of pulse profile computations}

We have performed several hundred million pulse profile computations, which we summarize below. Here we present results for an isotropically emitting spot of $25^{\circ}$ radius on a $1.4 \mathrm{M}_{\odot}$ reference star with circumferential radius of $R=5 G M / c^{2}(10.36 \mathrm{~km})$ and a spin rate of $400 \mathrm{~Hz}$ as seen at infinity. We also discuss the effects of deviations from these values. We consider emission from a single spot, two antipodal spots, and two spots at the same latitude but separated in azimuth by $160^{\circ}$. Although these computations were performed with APMXPs in mind, they can also be used to describe the oscillations produced by X-ray burst emission from a spot on the stellar surface.

\subsection{Persintently detected oscillations}

Figure 1 shows how the fractional amplitude produced by a single spot varies with the inclinations of the observer and the spot. Unless all APMXPs are observed at inclinations less than $10^{\circ}$, which is highly improbable, the small pulse amplitudes $(\sim 2 \%)$ that are observed occasionally in essentially all APMXPs are possible only if the spot is within $\sim 15^{\circ}$ of the spin pole. Amplitudes as high or higher than 50\% have not been observed from any APMXP. This behavior is natural in the nearly aligned moving spot model. The emitting spots would be nearly aligned if a star's magnetic poles move toward its spin axis as the star spins up [5].

The nearly aligned moving spot model also explains how the amplitudes of APMXP pulsations can vary from as low as $2 \%$ to as high as $19 \%$ within a few hours. Changes in the accretion flow can cause the centroid of the emitting region to move several degrees further from the spin pole on this timescale, increasing the pulsation amplitude by a substantial factor (see Figure 1). This model can also explain the observed changes in the arrival times of pulses by up to $\sim 0.3$ cycles on short timescales. Small displacements of a spot that is near the spin pole can easily produce changes in the arrival time this large. In contrast, a spot that is much farther from the spin pole would have to 


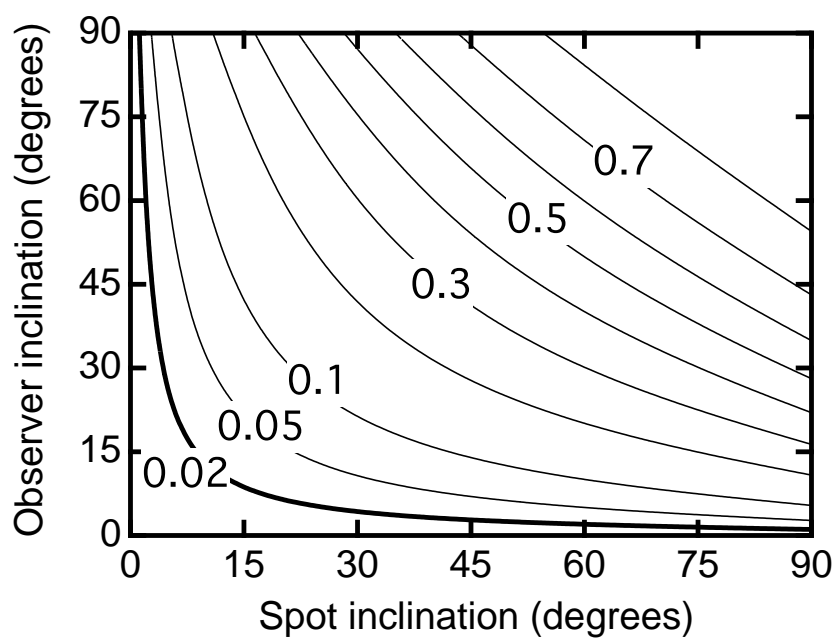

Figure 1: Contours showing the fractional amplitude of the fundamental component of the pulse profile produced by a single spot on our reference star, as a function of the observer and spot inclinations.

move $\sim 10$ kilometers in order to change the pulse arrival time by a comparable amount. Finally, spots at small inclinations naturally produce nearly sinusoidal pulse profiles like those observed.

The results for two spots at similar latitudes but separated in azimuth by $\sim 160^{\circ}-180^{\circ}$ are similar to the results for a single spot. The small observed amplitudes of the first overtone components of APMXP pulse profiles restrict the inclination of antipodal spots to small values. These results depend only weakly on the size of the spot and the compactness and spin frequency of the star. Our simulations show that the results are qualitatively similar for other radiation beaming patterns. See [8] for details.

\subsection{Intermittently detected oscillations}

Emitting regions that are close to the spin axis can also explain naturally how accretionpowered oscillations can appear and disappear on short timescales. As Figure 2 shows, movement of a spot center by a small amount, e.g., from $2^{\circ}$ to $8^{\circ}$, can cause the pulsations seen by an observer inclined at, e.g., $60^{\circ}$, to change from being undetectable by RXTE to being easily detectable, with an amplitude of $3 \%$. In this example, the first overtone would remain undetectable. Behavior like this occurs naturally for a variety of spot geometries and beaming patterns, and a wide range of spot radii, stellar masses and radii, and observer inclinations, provided the emitting region is close to the spin axis.

Changes in accretion rate are expected in connection with thermonuclear X-ray bursts, which could explain the apparent association in time of X-ray bursts and intermittent accretion-powered oscillations. The nearly aligned moving spot model also suggests the possibility of pulsation dropouts: when the accretion rate changes, the pulsing of an APMXP that typically produces lowamplitude pulsations may temporarily become undetectable. See [9] for details.

\subsection{Undetected oscillations}

The nearly-aligned moving spot model may also explain why accretion-powered X-ray pulsations have been detected in so few accreting neutron stars that are thought to have millisecond 


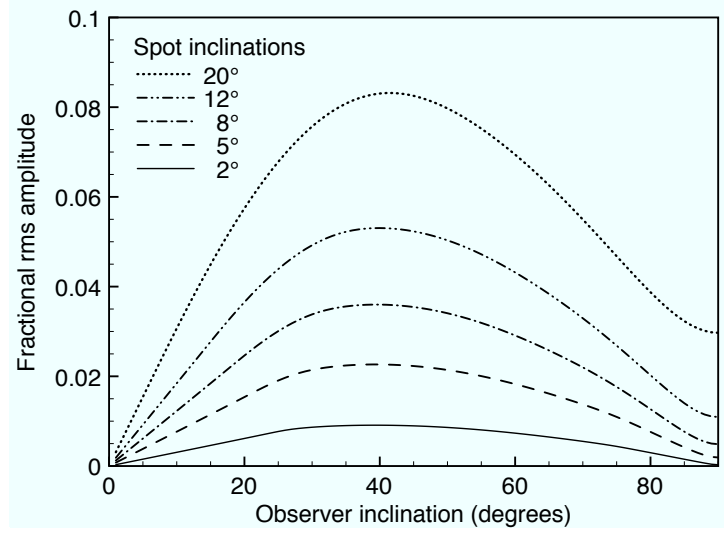

Figure 2: Fractional rms amplitude of the fundamental component of the pulse profile produced by antipodal spots on our reference star as a function of the observer inclination, for various small spot inclinations.

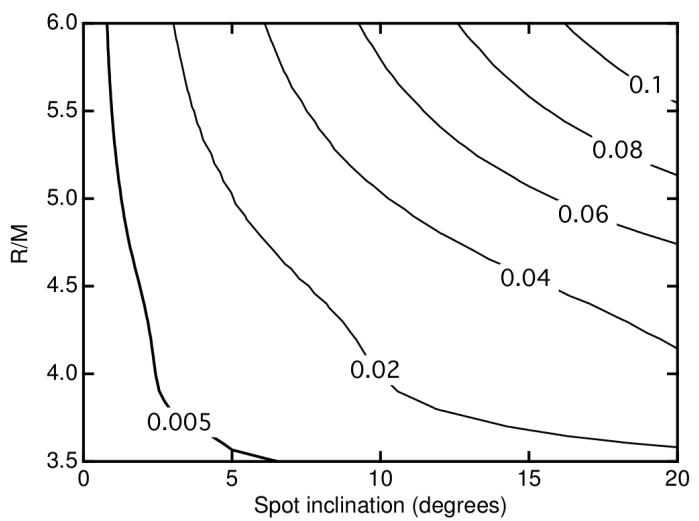

Figure 3: Contours showing the fractional amplitude of the fundamental component of the pulse profile produced by antipodal spots on our reference star, as a function of stellar compactness and spot inclination.

spin periods and magnetic fields strong enough to channel the accretion flow, at least to some extent. The radiation pattern of a spinning neutron star cannot be perfectly axisymmetric and such a star must therefore produce pulsations at some level. If, however, the emitting area is very close to the spin axis and remains there, the amplitude of the pulsations may be so low that they are undetectable with current instruments (see Figure 3).

The range of inclinations that produce pulsations too weak to be detected using current instruments is likely to be larger than the range indicated by Figure 3. The reason is that when the emitting area is near the spin axis, its azimuthal motion tends to produce large phase variations that reduce the amplitude that is obtained when the waveform is averaged by folding or by Fourier transforming the X-ray flux time series. Motions of the emitting area on timescales shorter than the interval used to construct a pulse profile can nevertheless be detected indirectly, because they will produce excess background noise in the power spectrum. The strength of this excess noise should be correlated with the amplitude and harmonic content of the pulse profile. Motions of the emitting area on timescales longer than the intervals of data used to construct pulse profiles can be inferred directly from the correlated changes in the amplitude and harmonic content of the pulse profile that they produce.

\section{Acknowledgments}

These results are based on research supported by a Humboldt Foundation fellowship at Tübingen, by NASA grant NAG 5-12030, NSF grant AST0709015, and funds of the Fortner Endowed Chair at Illinois, and by NSF grant AST0708424 at Maryland.

\section{References}

[1] Altamirano, D., Watts, A., Linares, M., Markwart, C. B., Strohmayer, T., Patruno, A., Type I $X$-ray bursts and burst oscillations in the accreting millisecond X-ray pulsar IGR J17511-3057, MNRAS 4091136 (2010) [arxiv: 1005 . 5299] 
[2] Altamirano, D., et al., Discovery of an accreting millisecond pulsar in the eclipsing binary system SWIFT J1749.4-2807, ApJ 72718 (2011) [arxiv: 1005.3527]

[3] Cadeau, C., Morsink, S. M., Leahy, D., \& Campbell, S. S., Light curves for rapidly rotating neutron stars, ApJ 654458 (2007) [astro-ph/ 0609325 ]

[4] Casella, P., Altamirano, D., Patruno, A., Wijnands, R., \& van der Klis, M., Intermittent millisecond X-ray pulsations from the neutron star X-ray transient SAX J1748.9-2021 in the globular cluster NGC 6440, ApJ 674 L41 (2008) [arxiv: 0708 . 1316]

[5] Chen, K., Ruderman, M., \& Zhu, T., Millisecond pulsar alignment: PSR J0437-4715, ApJ 493397 (1998)

[6] Galloway, D. K., Morgan, E. H., Krauss, M. I., Kaaret, P., \& Chakrabarty, D., Intermittent pulsations in an accretion-powered millisecond pulsar, ApJ 65473 (2007) [astro-ph/0609693]

[7] Lamb, F. K., \& Boutloukos, S., Accreting neutron stars in low-mass X-ray binary systems, in Short-period binary stars, Ap. \& Space Sci. Library 35287 (2008) [arXiv: 0705 . 015]

[8] Lamb, F. K., Boutloukos, S., Van Wassenhove, S., Chamberlain, R. T., Lo, K. H., Clare, A., Yu, W., \& Miller, M. C., A model for the waveform behavior of accreting millisecond X-ray pulsars: Nearly aligned magnetic fields and moving emission regions, ApJ 706417 (2009b) [arxiv:0808.4159]

[9] Lamb, F. K., Boutloukos, S., Van Wassenhove, S., Chamberlain, R. T., Lo, K. H., \& Miller, M. C., Origin of intermittent accretion-powered X-ray oscillations in neutron stars with millisecond spin periods, ApJ, 70536 (2009a) [arxiv: 0809 . 4016]

[10] Liu, Q. Z., van Paradijs, J., \& van den Heuvel, E. P. J., A catalogue of low-mass X-ray binaries in the Galaxy, LMC, and SMC, A\&A 46980 (2007) [arxiv: 0707.054 4]

[11] Miller, M. C., \& Lamb, F. K., Bounds on the compactness of neutron stars from brightness oscillations during X-Ray burst, ApJ 499 L37 (1998) [ast ro-ph/9711325]

[12] Patruno, A., Searching sub-millisecond pulsars in accreting neutron stars, in High time resolution astrophysics IV, POS (HTRA-IV) 028 (2010) [arXiv: 1007.1108 ]

[13] Patruno, A., Hartman, J. M., Wijnands, R., Chakrabarty, D., \& van der Klis, M., Accretion torques and motion of the hot spot on the accreting millisecond pulsar XTE J1807-294, ApJ 7171253 (2010) [arxiv:0902.4323]

[14] Patruno, A., Wijnands, R., \& van der Klis, M., An alternative interpretation of the timing noise in accreting millisecond pulsars, ApJ 69860 (2009) [arXiv: 0904 .2383]

[15] Wijnands, R., Accretion-driven millisecond X-ray pulsars, in Trends in pulsar research, Nova Science, 53 (2006) [ast ro-ph/0501264] 